\title{
GRINDING METHOD, TRAJECTORY PLANNING AND SIMULATION OF A 3 DOF KNEE GRINDING ROBOT
}

\author{
Tian, H. ${ }^{*}$, ; Ma, L. ${ }^{*}$; Zhu, X. ${ }^{*} \&$ Dang, X. ${ }^{* *}$ \\ *College of Mechanical and Electronic Engineering, Shandong University of Science and Technology, \\ Qingdao 266590, China \\ ** Qingdao Municipal Hospital Group, Qingdao 266073, China \\ E-Mail: tianhq26@sdust.edu.cn, dxqcys0518@163.com (" Corresponding author)
}

\begin{abstract}
This paper aims to improve the accuracy of robot-assisted grinding in total knee arthroplasty (TKA). For this purpose, a 3 DOF knee grinding robot was designed, combining the merits of series robot and parallel robot. The kinematics equations of the robot were derived by the vector method and the workspace of the robot was identified through Monte-Carlo method. Then, the grinding method of the robot was developed according to the procedure of the TKA. Specifically, the grinding trajectory was planned considering the implant selected for the patient. First, the plane and boundary equations of the implant were determined; then, the femur was ground into the desired shape; after that, the implant was cut off with a ball cutting tool vertically to the depth equal to the row spacing. To validate the trajectory planning method, a simulation analysis was performed on the planned trajectory. The results prove the feasibility and efficiency of the proposed method. The research findings shed new light on the robot-assisted TKA.

(Received, processed and accepted by the Chinese Representative Office.)
\end{abstract}

Key Words: Knee Grinding Robot, Kinematics, Workspace, Grinding Method, Trajectory Planning

\section{INTRODUCTION}

At present, orthopaedic surgery has become a leading application field of medical robotics. For example, bone grinding robots have been successfully implemented in total hip arthroplasty (THA) and total knee arthroplasty (TKA), yielding better results than the conventional methods. Below is a brief review of some representative bone grinding robots.

The Robodoc surgical robot [1] can properly reshape the femoral section for the placement of the implant according to the planned path. The Caspar robot [2] boasts a grinding accuracy of $0.1 \mathrm{~mm}$ in both THA and TKA. The semi-active Acrobot surgical system [3] supports bone cutting in the TKA and the minimally invasive knee arthroplasty, and acquires the real-time feedback of cutting force through a driven-end device. The RIO robotic arm system mainly performs bone grinding in the TKA [4, 5] with force feedbacks and minimal damages. Designed for the THA, the Arthorobot system [6] can be mounted on bones and controlled directly by doctors through the master-slave mode. The MBARS system [7] is also mounted on the bones for patellofemoral arthroplasty (PFA). It can complete bone cutting in $2 \mathrm{~min}$, which is faster than manual cutting. Some researchers have developed surgical robots with a hybrid structure, which combines the merits of both serial and parallel structures. For instance, Bruni et al. [8] invented a series-parallel hybrid robot for the TKA, in which the mechanical body uses a series structure and the processing head adopts a parallel structure. Song et al. [9] created a series-parallel hybrid orthopaedic robot HyBAR for joint replacement surgeries.

In addition, much research has been done on the trajectory planning of bone grinding robots. Abraham et al. [10] put forward an automatic calculation algorithm for robot's grinding trajectory in Cartesian space, which covers the entire bone surface with element decomposition, scans lines and potential functions, and successfully applies it in MBARS and 
HyBAR systems. Guven and Barkana [11] define the bone grinding trajectory in the image space and converts it into a robotic bone space for bone tissue grinding. Sugita et al. [12] present a trajectory generation strategy based on geometric model and implemented it in a 7axis grinding robot, revealing that the strategy can prevent soft tissue damage. Fan et al. [13] propose a parallel grinding and non-interfering trajectory planning method for implant grinding robot, which prevents the interference between the two grinding trajectories, and introduce the quadtree theory to speed up the generation of tool trajectory. Tian et al. [14] develop a trajectory generation approach for parameterized modular grinding based on the features of cervical disc replacement and the structure of cervical vertebrae and minimize the damage to the patient through surface and spherical grinding of the implant. Federspil et al. [15] explore the skeletal grinding method of ear neurosurgery and prove the correctness and effectiveness of this method through grinding experiments.

In the TKA, grinding trajectory planning determines whether the ground femoral surface matches the knee joint implant, and thus the physiological function of the patient's knee. According to the femur requirements of the knee joint, this paper looks for a suitable femoral trajectory planning method and determines the suitable position for knee joint implant through the implementation of this planning method.

\section{KINEMATICS AND WORKSPACE OF KNEE GRINDING ROBOT}

\subsection{Structural design}

In view of its functions and requirements, the knee joint grinding robot was analysed in terms of structural composition, DOFs and driving methods. Here, a series-parallel hybrid structure is adopted and mapped to a cylindrical coordinate system. The robot consists of a planar parallel mechanism and a vertical mechanism. Thus, it mainly moves reciprocally in the plane direction and the vertical direction. The reciprocating motion in the plane direction enables the medical drill to reach the proper working position. Despite the changes to the angle between the two mechanisms, the rigid triangular structure is maintained with the connecting member, eliminating the eccentricity of the drill. The reciprocating motion in the vertical direction allows the drill to return to the initial position after the surgery. To complete the above motions, the robot is driven by an auto-fed nut drive. The vertical mechanism is a vertically-arranged double-track single-spiral slide with an effective stroke of $170 \mathrm{~mm}$, while the planar parallel mechanism is a horizontally-arranged double-track single-spiral slide with an effective stroke of $170 \mathrm{~mm}$.According to the relevant parameters, the planar parallel mechanism is driven by a Maxon RE-16 motor (rated power: $4.5 \mathrm{~W}$; rated voltage: $4.8 \mathrm{~V}$; rated torque: $2.15 \mathrm{Nm}$; rated speed: $12,700 \mathrm{rpm}$ ) with a $261: 1$ reducer; the vertical mechanism is driven by a Maxon EC22 motor (rated power: $40 \mathrm{~W}$; rated torque: $15.2 \mathrm{Nm}$; rated speed: 30,800 rpm).

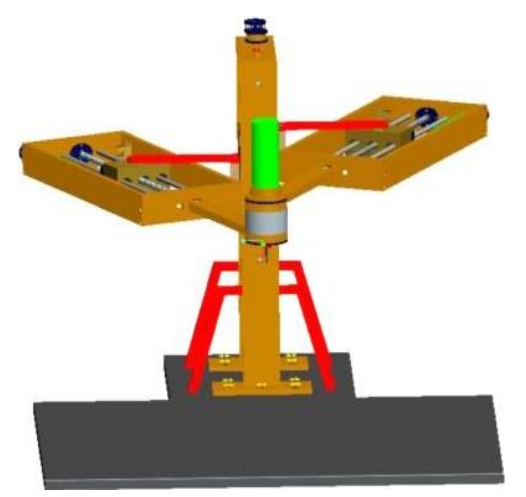

Figure 1: 3 DOF knee joint grinding robot. 
As shown in Fig. 1, the established model for knee joint grinding robot has a 3 DOF series-parallel hybrid structure. One DOF refers to the series-wound vertical mechanism, which achieves the vertical movement of the whole body; the other two DOFs refer to the planar parallel mechanism and the synthesis between the plane and vertical mechanisms; together, the latter two DOFs realize the plane movement in the endoscope plane.

\subsection{Kinematics}

The vector method was adopted to solve the inverse kinematics position solution of the robot. The relationship between the point $\mathrm{C}$ of the end effector $\left(x_{c}, y_{c}, z_{c}\right)$ and the fixed coordinate system $O(x, y, z)$ can be expressed by the vector, and the length change of each member of the robot can be derived according to the relationship (Figs. 2 and 3).

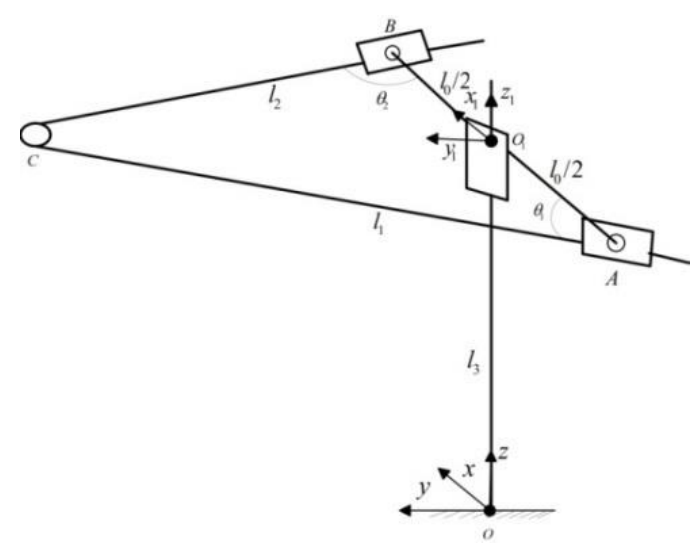

Figure 2: Schematic diagram of knee joint grinding robot.

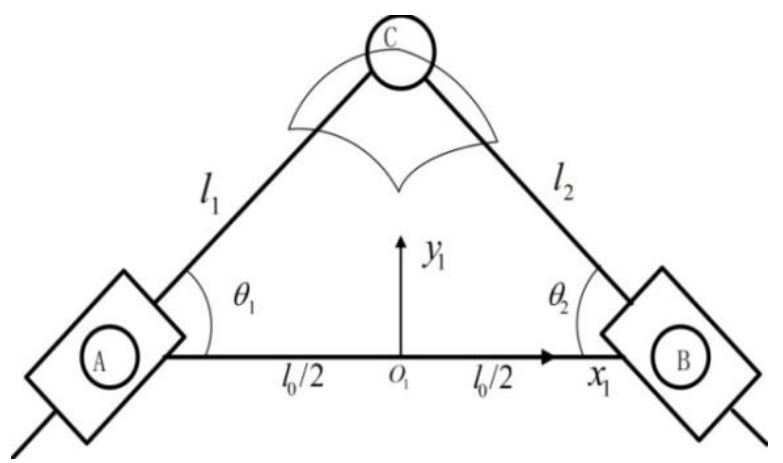

Figure 3: Schematic diagram of robot parallel part.

(1) Inverse kinematics

The coordinates of the end effector $\mathrm{C}$ in $O(x, y, z)$ in Fig. 2 can be expressed as:

$$
\left\{\begin{array}{l}
x_{c}=-\frac{l_{0}}{2}+\cos \theta_{1} \cdot l_{1} \\
y_{c}=\sin \theta_{1} \cdot l_{1} \\
z_{C}=o_{1 Z}=l_{3}
\end{array}\right.
$$

The coordinates of the end effector $\mathrm{C}$ in $O(x, y, z)$ in Fig. 2 can also be expressed as:

$$
\left\{\begin{array}{l}
x_{c}=\frac{l_{0}}{2}-\cos \theta_{2} \cdot l_{2} \\
y_{c}=\sin \theta_{2} \cdot l_{2} \\
z_{C}=o_{1 Z}=l_{3}
\end{array}\right.
$$

By Eqs. (1) and (2), we can get:

$$
\begin{aligned}
& l_{1}=\sqrt{x_{c}{ }^{2}+y_{c}{ }^{2}+\frac{l_{0}^{2}}{4}+l_{0} \cdot x_{c}} \\
& l_{2}=\sqrt{x_{c}{ }^{2}+y_{c}^{2}+\frac{l_{0}^{2}}{4}-l_{0} \cdot x_{c}}
\end{aligned}
$$

Thus, the $z$ value of knee joint $l_{3}$ of the grinding robot in Fig. 2 can be obtained as: 


$$
l_{3}=z_{C}=z_{O 1}
$$

Using the above equation, the inverse kinematics equation of the robot can be solved as:

$$
\left\{\begin{array}{l}
\Delta l_{1}=\sqrt{x_{c}{ }^{2}+y_{c}{ }^{2}+\frac{l_{0}^{2}}{4}+l_{0} \cdot x_{c}}-l_{1}^{\prime} \\
\Delta l_{2}=\sqrt{x_{c}{ }^{2}+y_{c}{ }^{2}+\frac{l_{0}^{2}}{4}-l_{0} \cdot x_{c}}-l_{2}^{\prime} \\
\Delta l_{3}=z_{c}-l_{3}^{\prime}
\end{array}\right.
$$

where $l_{1}^{\prime}, l_{2}^{\prime}$ and $l_{3}^{\prime}$ are the length of the members at the initial position.

(2) Forward kinematics

To obtain the forward kinematics equation of the robot, the position of the end effector in the workspace of the robot can be determined under the known movement of each member. The variation of $l_{1}^{\prime}, l_{2}^{\prime}$ and $l_{3}^{\prime}$ is equivalent to the change of the end effector point $C\left(x_{c}, y_{c}, z_{c}\right)$ in $O(x, y, z)$. The robot parallel part is a triangular structure that changes with the $l_{1}^{\prime}$ and $l_{2}^{\prime}$ in the grinding process. As shown in Fig. 3, the variation can be viewed as the movements of point $\mathrm{C}$ with the circle centred on point $\mathrm{A}$ and the circle centred on point $\mathrm{B}$.

In the fixed coordinate system $O(x, y, z)$, the coordinates of points $\mathrm{A}$ and $\mathrm{B}$ are $A\left(-l_{0} / 2,0\right.$, $\left.l_{3}\right)$ and $B\left(-l_{0} / 2,0, l_{3}\right)$, respectively. Thus, there exist the following constraints:

$$
\left\{\begin{array}{l}
\left(x_{c}+\frac{l_{0}}{2}\right)^{2}+y_{c}{ }^{2}=l_{1}^{2} \\
\left(x_{c}-\frac{l_{0}}{2}\right)^{2}+y_{c}{ }^{2}=l_{2}^{2}
\end{array}\right.
$$

The forward kinematics solution of the robot can be obtained from the above equation, which is the variation of $C\left(x_{c}, y_{c}, z_{c}\right)$ in $O_{1}\left(x_{1}, y_{1}, z_{1}\right)$ :

$$
\left\{\begin{array}{l}
x_{c}=\frac{l_{1}^{2}-l_{2}^{2}}{2 l_{0}} \\
y_{c}=\sqrt{\frac{l_{1}^{2}}{2}-\frac{l_{0}^{2}}{4}+\frac{l_{2}^{2}}{2}-\frac{\left(l_{1}^{2}-l_{2}^{2}\right)^{2}}{4 l_{0}^{2}}}
\end{array}\right.
$$

From Fig. 2, it can be seen that point $\mathrm{O}_{1}$ and point $\mathrm{C}$ in the coordinate system $O(x, y, z)$ share the same $z$ value: $Z_{C}=Z_{O 1}=l_{3}$.

Hence, the $\mathrm{z}$ value of point $\mathrm{C}$ of the robot in the fixed coordinate system $O(x, y, z)$ can be expressed as:

$$
\left\{\begin{array}{l}
x_{c}=\frac{l_{1}^{2}-l_{2}^{2}}{2 l_{0}} \\
y_{c}=\sqrt{\frac{l_{1}^{2}}{2}-\frac{l_{0}^{2}}{4}+\frac{l_{2}^{2}}{2}-\frac{\left(l_{1}^{2}-l_{2}^{2}\right)^{2}}{4 l_{0}^{2}}} \\
z_{C}=l_{3}
\end{array}\right.
$$

\subsection{Workspace}

To determine the workspace of the robot within the constraints on joint change, the 3D coordinates of the end effector was simulated according to the kinematics solution through 
Matlab programming and Monte-Carlo method. The Monte-Carlo method is a simple and popular approach that solves the workspace by generalizing joint variables through random sampling. A total of 10,000 points were extracted from the workspace shown in Figs. 4 to 7. According to the results of the Monte-Carlo method, the workspace is probably cylindrical under the constraints on rod length $157 \mathrm{~mm} \leq l_{1} \leq 267 \mathrm{~mm}, 157 \mathrm{~mm} \leq l_{2} \leq 267 \mathrm{~mm}$ and $78 \mathrm{~mm} \leq l_{3} \leq 182 \mathrm{~mm}$; the $x, y$ and $z$ respectively fall in the ranges of $-80 \mathrm{~mm} \leq x \leq 80 \mathrm{~mm}$, $80 \mathrm{~mm} \leq y \leq 240 \mathrm{~mm}$ and $80 \mathrm{~mm} \leq z \leq 180 \mathrm{~mm}$; the size of the workspace is roughly $11 \times 10 \times 10 \mathrm{~mm}^{3}$ relative to the size range of human knee joint. Suffice it to say that the workspace is sufficiently large for the grinding operation.

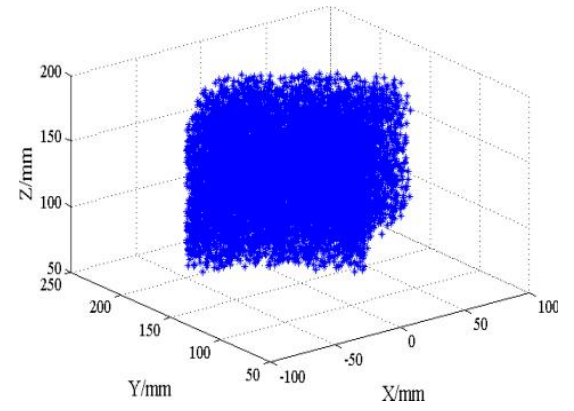

Figure 4: Full 3D workspace.

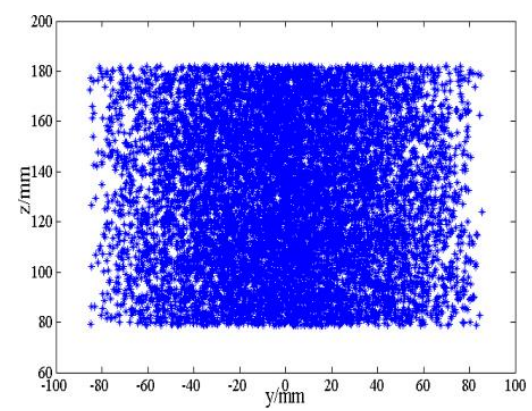

Figure 6: Workspace projection on ZY plane.

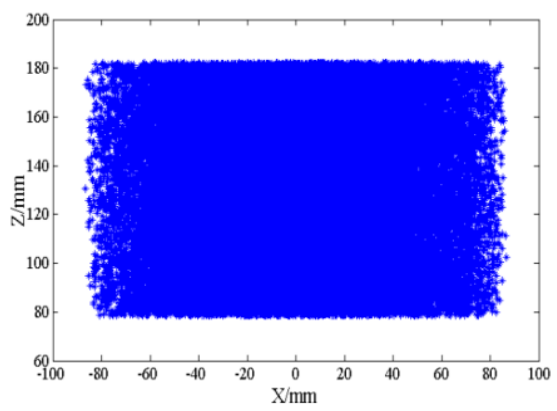

Figure 5: Workspace projection on the XZ plane.

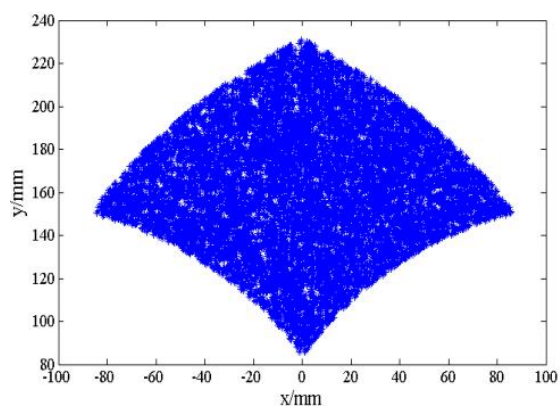

Figure 7: Workspace projection on XY plane.

\section{GRINDING METHOD OF KNEE GRINDING ROBOT}

\subsection{The TKA process}

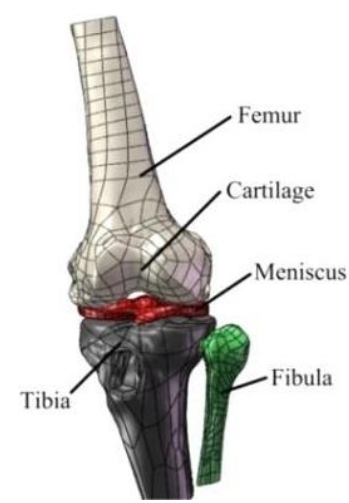

Figure 8: 3D model of knee joint.

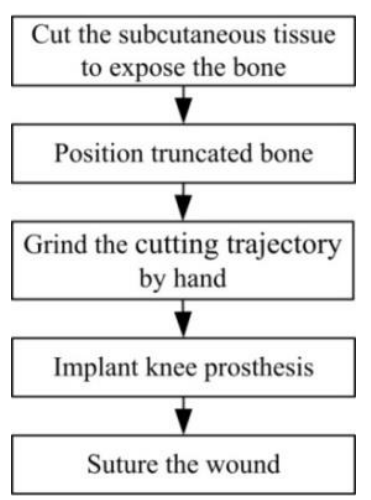

Figure 9: Procedure of the TKA.

As shown in Fig. 8, the knee bone involves the following main components: femur, cartilage, meniscus, tibia and fibula. The meniscus lies between the femur and tibia. The tibia is attached to the femur through the cartilage of the trochlear. On the surface of the femur, the cartilage is scalloped and has a certain service life. Cartilage damage might occur after year- 
around frictions. The 3DOF knee grinding robot is mainly designed to grind the injured cartilage into an ideal shape for the insertion of the implant. The procedure of the TKA is explained in Fig. 9. The conventional method relies heavily on numerous bone markers as a reference for positioning and osteotomy. In traditional TKA, the placement of implant depends on the mastery of related techniques, and the femoral surface is rarely ground to the shape that matches the implant perfectly. To solve the problem, the knee joint grinding robot has been introduced to grind the femur surface and implant surface.

\subsection{Robot-assisted grinding method}

The main workflow of robot-assisted grinding method is as follows:

(1) Perform a layer-by-layer CT scan of the knee joint and use mathematical methods and computer imaging to gather the 3D information of the knee joint.

(2) Conduct digital processing of the knee joint in Mimics, draw the fitting curves of the joint in Geomagic and build the model of the joint in UG.

(3) Select the appropriate implant from the implant pool according to the knee joint lesions of the patient.

(4) Determine the relationship between the femoral and knee joint in the fixed coordinate system, that is, calibrate the robot coordinate system and the femoral coordinate system and determine the start point of the grinding (Fig. 10).

(5) Plan the grinding trajectory based on the surface and boundary equations of the implant and the cut-off line equation.

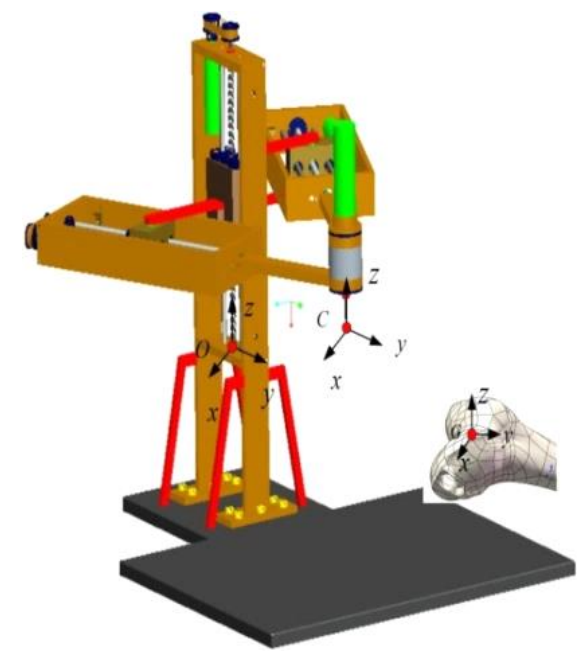

Figure 10: Calibration of coordinate system for grinding start point.

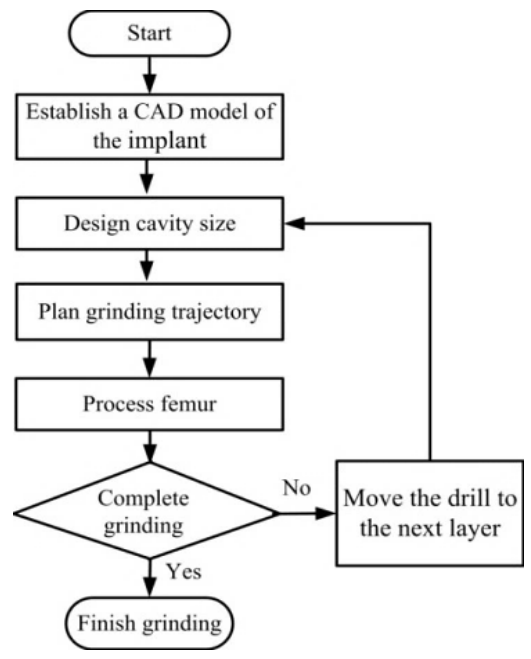

Figure 11: Grinding planning process.

\section{TRAJECTORY PLANNING OF KNEE GRINDING ROBOT}

In the TKA, the lower surface centreline of the implant must match the groove on the trochlear cartilage surface, while the upper surface centreline of the implant must fall on the same plane with the tibia centreline. The distance of the implant must be far, and the end and proximal ends must be parallel to the surface of the femur cartilage. The grooves on the trochlear surface and the corresponding points on the implant centreline should be marked by software. During the operation, the knee joint of the patient was scanned by CT and simulated in 3D modelling software. According to the lesions of the model, a CAD model with a scallop-shaped implant was selected from the implant bank. After the trajectory and position were calibrated, the grinding depth, rotation speed and feed amount were set and the femur was ground with the drill in the vertical posture. Then, the contour of the bone cavity was 
ground to match the contour of the selected implant along a proper trajectory. Considering the thickness of the implant, the grinding was carried out layer by layer (Fig. 11).

Specifically, the trajectory planning involves the following steps:

(1) Determine implant specifications

After setting up the 3D model for the knee of the patient, the lesions of the knee were observed carefully, and then the implant pool was searched to find the implant that matches the shape and size of the knee (Fig. 12).

(2) Determine the equations of grinding boundaries and surface

The selected implant has a scallop-like structure. The two intersecting planes are shown in Fig. 13, and the boundary curves are displayed in Fig. 14.

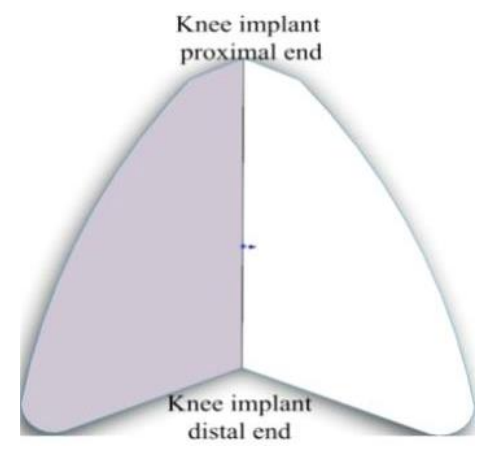

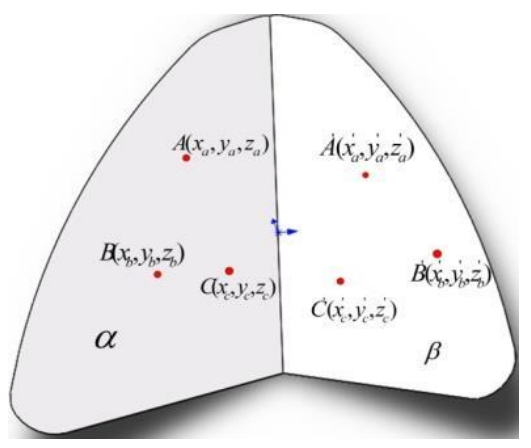

Figure 12: Implant of knee joint. Figure 13: Planes of the implant. Figure 14: Boundary curves of the implant.

In the coordinate system of the implant $M(x, y, z)$, three random points $A\left(x_{a}, y_{a}, z_{a}\right)$, $B\left(x_{b}, y_{b}, z_{b}\right)$ and $C\left(x_{c}, y_{c}, z_{c}\right)$ were selected from plane $\alpha$ and another three $A^{\prime}\left(x_{a}^{\prime}, y_{a}^{\prime}, z_{a}^{\prime}\right)$, $B^{\prime}\left(x_{b}^{\prime}, y_{b}^{\prime}, z_{b}^{\prime}\right)$ and $C^{\prime}\left(x_{c}^{\prime}, y_{c}^{\prime}, z_{c}^{\prime}\right)$ were selected from plane $\beta$ :

$$
\begin{aligned}
& A_{1} x+B_{1} y+C_{1} z+D_{1}=0 \\
& A_{2} x+B_{2} y+C_{2} z+D_{2}=0
\end{aligned}
$$

After fitting the two planes to the implant, the boundary constraints were determined one by one. First, the 3D coordinates of the implant were inputted to ANSYS; then, the surface was divided into four constraint boundary curves, denoted as 1, 2, 3 and 4, respectively; meanwhile, the knee surface was divided into another four constraint boundary curves, denoted as 4, 5, 6 and 7, respectively. Using the $1^{\text {st }}$ Opt fitting algorithm, the curves $1,2,6$ and 7 were simplified as an arc for further processing.

For any three points $\left(x_{1}, y_{1}, z_{1}\right),\left(x_{2}, y_{2}, z_{2}\right)$ and $\left(x_{3}, y_{3}, z_{3}\right)$ on the arc, the distance from each point to the centre coordinates $(x, y, z)$ should satisfy the condition in Eqs. (10) and (12):

$$
\left\{\begin{array}{l}
R^{2}=\left(x_{1}-x\right)^{2}+\left(y_{1}-y\right)^{2}+\left(z_{1}-z\right)^{2} \\
R^{2}=\left(x_{2}-x\right)^{2}+\left(y_{2}-y\right)^{2}+\left(z_{2}-z\right)^{2} \\
R^{2}=\left(x_{3}-x\right)^{2}+\left(y_{3}-y\right)^{2}+\left(z_{3}-z\right)^{2}
\end{array}\right.
$$

The above equation should be simplified to eliminate $R$ :

$$
\begin{aligned}
& A_{3} x+B_{3} y+C_{3} z+D_{3}=0 \\
& A_{4} x+B_{4} y+C_{4} z+D_{4}=0
\end{aligned}
$$

The matrix form of Eq. (10) can be expressed as:

$$
\left[\begin{array}{lll}
A_{1} & B_{1} & C_{1} \\
A_{3} & B_{3} & C_{3} \\
A_{4} & B_{4} & C_{4}
\end{array}\right]\left[\begin{array}{l}
x \\
y \\
z
\end{array}\right]+\left[\begin{array}{c}
D_{1} \\
D_{3} \\
D_{4}
\end{array}\right]=0
$$


(3) Set grinding parameters

The designed robot uses a ball cutting tool as the cutter. As shown in Fig. 15 a, the centre point $C$ of the end effector is the centre point of the cutting tool [10]. The grinding parameters should be selected in light of the particularity of the grinding material, including rotation speed $n(\mathrm{r} / \mathrm{min})$, grinding depth $a_{p o}(\mathrm{~mm})$, row spacing $a_{e o}(\mathrm{~mm})$, feed rate $f_{z}(\mathrm{~mm} / \mathrm{z})$, ball nose radius $R(\mathrm{~mm})$, feed speed $v_{f}(\mathrm{~mm} / \mathrm{min})$, cutter teeth $z$ and burr height $h$. Note that the burr height is related to the grinding depth and grinding speed. To ensure the match between the joint surface and implant surface, both surfaces should be polished to control the burr height and satisfy $a_{p o}<2 R$.

The smoothness of the polished surfaces hinges on the properness of the row spacing. The smaller the row spacing, the more likely it is for the burr height to fall below the cutter radius. Hence, the row spacing should be reduced continuously until the surfaces are nearly smooth. Considering the transmission mechanism of the robot, large stroke movements should be avoided to reduce the error arising from transmission gap.

In actual processing, the burr height $h$ should be controlled to ensure the match between the knee joint and the implant. As shown in Fig. $15 \mathrm{~b}$, the burr height $h$ and row spacing $a_{e o}$ can be determined by Eq. (16) below.

$$
(R-h)^{2}+\left(\frac{a_{e o}}{2}\right)^{2}=R^{2}
$$

where $h$ is the burr height; $R$ is the cutter radius; $a_{e o}$ is the row spacing.

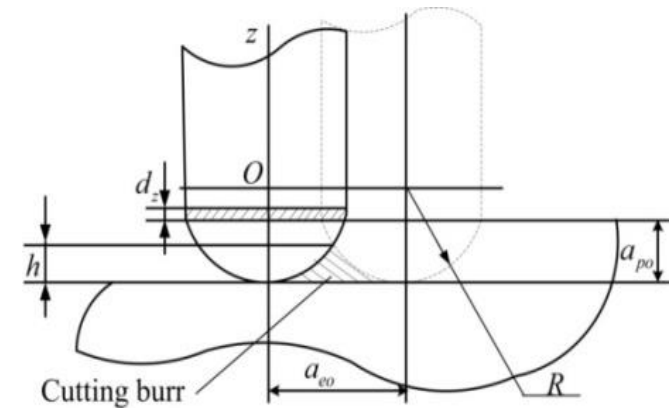

(a)

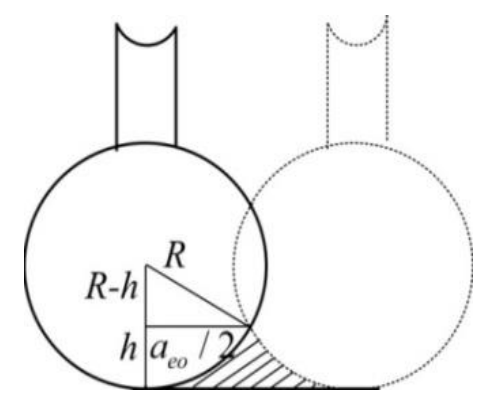

(b)

Figure 15: Schematic diagram of bone grinding with a ball cutting tool.

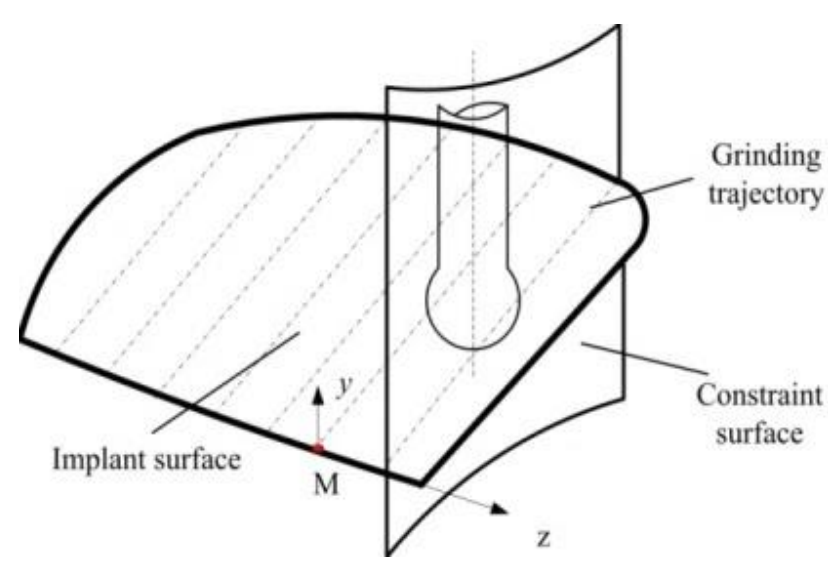

Figure 16: Grinding trajectory planning based on cross-section method.

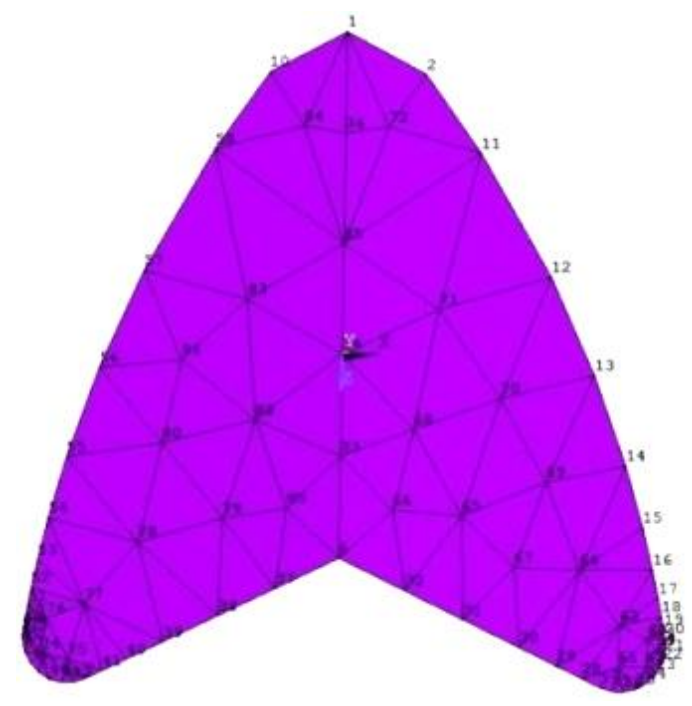

Figure 17: Implant mesh model of knee joint. 
(4) Plan grinding trajectory

As mentioned before, the implant was selected based on the lesions of the patient's knee joint and compose of two scallop-shaped surfaces and boundary curves. The grinding trajectory was rationally planned according to the shape of the implant surface. Due to the limited flexibility of the robot, the $3 \mathrm{DOF}$ end effector has no pose. In actual operation, the grinding trajectory should be planned based on the coordinates of the proximal and distal parts of the implant in the coordinate system M. Here, the grinding trajectory is planned by cross-section method. With the $a_{e o}$ plane perpendicular to the z-axis and spaced by one processing row, the selected implant was sequentially cut off (Fig. 16). The V-shaped cutter trajectory and the boundary curves on the joint surface constitute the constraints. In this way, the grinding trajectory was determined.

\section{CASE STUDY}

A scalloped implant with two intersection planes was selected according to the shape of the patient's knee joint. Then, the grinding trajectory was planned through plane fitting and boundary determination.

\subsection{Plane fitting}

The planes were fitted in the following steps: Import the implant model into ANSYS in the format of *.iges; after implant model meshing (Fig. 17 ), plot the grid points by Plot-Nodes and count the grid points; select three nonlinear points; then, fit the plane equation by Matlab.

Initially, 85 grid points (Table I) were obtained from the implant. The $3 \mathrm{D}$ coordinates of these points on mesh model in Fig. 20 are shown below. Then, three points were selected from plane $\alpha$ : A $(-8.2782,4.7794,-12.221)$, B $(-10.177,5.8759,0.57786)$ and $\mathrm{C}(-6.0809$, $3.5108,-3.1404)$, and three points from plane $\beta: A^{\prime}(8.2782,4.7794,-12.221), B^{\prime}(12.666$, $7.3125,-4.8505)$ and $C^{\prime}(5.8460,3.33752,-2.7268)$. Finally, fit the planes of each group of three points by Matlab (Figs. 18 and 19). To ensure the fitting accuracy, the planes were fitted several times to yield the mean values of the fitting coefficients $A_{1}, B_{1}$, and $C_{1}$. The fitting equations are as follows:

$$
\begin{aligned}
& 20.9531 \cdot x+36.2902 \cdot y-0.0010 \cdot z+0.0032=0 \\
& 19.8508 \cdot x-34.3831 \cdot y+0.0013 \cdot z+0.0019=0
\end{aligned}
$$

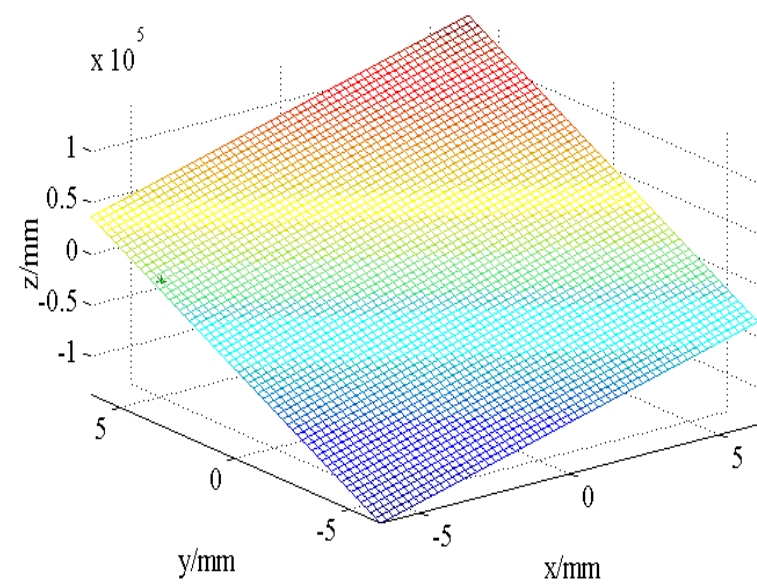

Figure 18: The plane of points A, B and C.

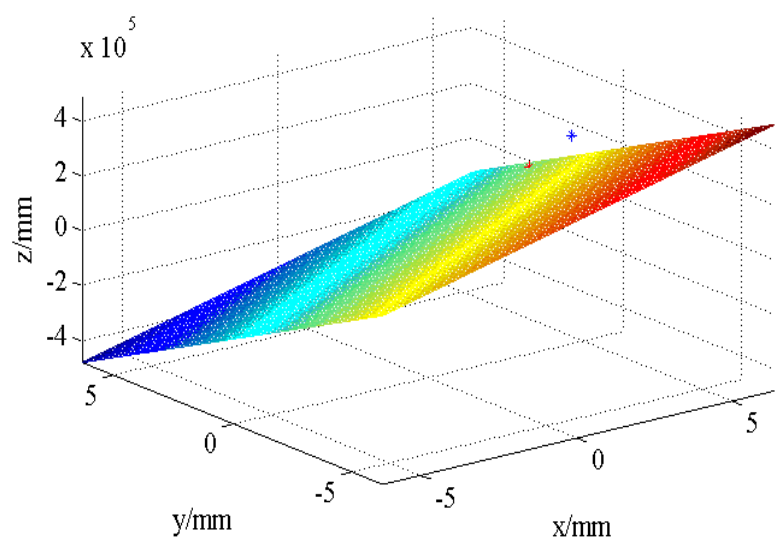

Figure 19: The plane of points $A^{\prime}, B^{\prime}$ and $C^{\prime}$. 
Table I: Data on the initial grid points on mesh model.

\begin{tabular}{|c|c|c|c|}
\hline \multirow{2}{*}{ NO. } & \multicolumn{3}{|c|}{ Coordinates of points } \\
\cline { 2 - 4 } & $\mathrm{X}$ & $\mathrm{Y}$ & $\mathrm{Z}$ \\
\hline 1 & 0.0000 & 0.0000 & -19.405 \\
\hline 2 & 4.8553 & 2.8032 & -16.912 \\
\hline 3 & 20.000 & 11.547 & 17.204 \\
\hline 4 & 20.000 & 11.547 & 17.550 \\
\hline 5 & $\ldots \ldots$ & $\ldots \ldots$ & $\ldots \ldots$ \\
\hline
\end{tabular}

\subsection{Determination of boundary conditions}

Table II: Boundary equations of the implant.

\begin{tabular}{|c|c|c|}
\hline Boundary type & Boundary equation & Boundary line diagram \\
\hline Arc 1 & $53.87^{2}=(x-38.56)^{2}+(y+22.26)^{2}+(z-30.33)^{2}$ & \\
\hline Arc 2 & $5.32^{2}=(x+17.72)^{2}+(y-10.23)^{2}+(z-17.27)^{2}$ & \\
\hline Arc 6 & $5.32^{2}=(x-17.72)^{2}+(y-10.23)^{2}+(z-17.27)^{2}$ & \\
\hline Arc 7 & $53.87^{2}=(x+38.56)^{2}+(y+22.26)^{2}+(z-30.33)^{2}$ & \\
\hline Line 3 & $\frac{x}{-13.373}=\frac{y}{7.7207}=\frac{z-12.433}{6.271}$ & \\
\hline Line 4 & $\left\{\begin{array}{l}x=0, y=0 \\
-19.45 \leq z \leq 12.433\end{array}\right.$ & \\
\hline Line 5 & $\frac{x}{13.373}=\frac{y}{7.7207}=\frac{z-12.433}{6.271}$ & $\sqrt{2}$ \\
\hline
\end{tabular}


After fitting the above two planes, the implant boundaries were determined (see Fig. 14). Specifically, the scatter points of each boundary were fitted into a curve, and the trajectory equation was plotted by Matlab within a proper coordinate range. The boundary conditions of these curves are written in Table II. Where, for the $1^{\text {st }}$ and $7^{\text {th }}$ curves, the curve shape is approximately an arc in a specific range; for the $2^{\text {nd }}$ and $6^{\text {th }}$ curves, the curve shape is also approximately an arc in a specific range; for the $3^{\text {rd }}$ and $5^{\text {th }}$ curves, the curve shape is a straight line; for the $4^{\text {th }}$ curve, the curve shape is a segment of the $\mathrm{z}$-axis. The implant model can be determined by combining the two fitted planes and the boundary conditions. To sum up, the boundaries of the $1^{\text {st }}, 2^{\text {nd }}, 6^{\text {th }}$ and $7^{\text {th }}$ curves are all circular arcs and the boundaries of the $3^{\text {rd }}, 4^{\text {th }}$ and $5^{\text {th }}$ curves are all straight lines.

\subsection{Trajectory planning}

The grinding trajectory was planned based on the selected implant. In the $\mathrm{M}$ coordinate system, the implant range in the $\mathrm{z}$-axis is $-19.405 \leq \mathrm{z} \leq 20.183$. Thus, the trajectory equation can be expressed as $z=-19.405+n_{i} \cdot \alpha_{e o}$, with $n_{i}$ being the number of cross-sections. The cutting of planes $\alpha$ and $\beta$ should start from the proximal end, leaving a $\mathrm{V}$-shaped trajectory on both planes. Set up the internal surface equation of the implant as follows (see Table III).

Table III: Internal surface equations of the implant.

\begin{tabular}{|c|c|c|}
\hline Implant plane & Plane trajectory equation & V-shaped cutting trajectory \\
\hline Plane $\alpha$ & $20.9531 \cdot x+36.2902 \cdot y-0.0010 n_{i} \cdot a_{e o}+0.02265=0$ & $\begin{array}{l}\text { Knes implant } \\
\text { proximal end }\end{array}$ \\
\hline Plane $\beta$ & $19.8508 \cdot x-34.3831 \cdot y+0.013 n_{i} \cdot a_{e o}-0.2503=0$ & 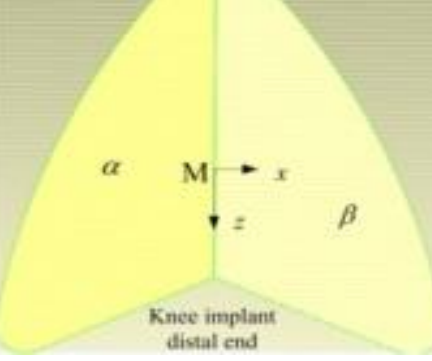 \\
\hline
\end{tabular}

\subsection{Trajectory simulation}

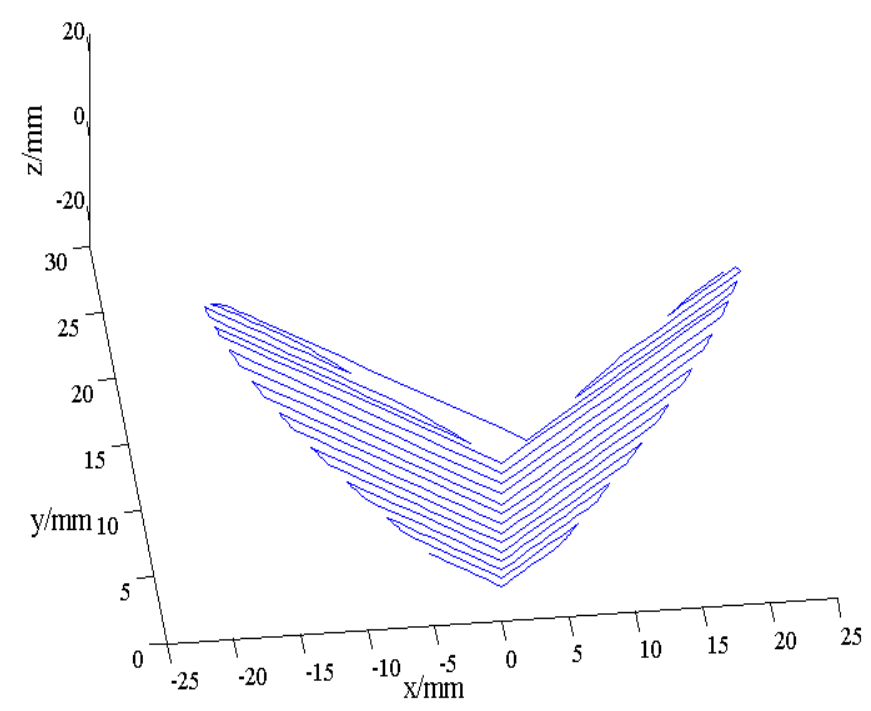

Figure 20: Internal V-shaped trajectory. 


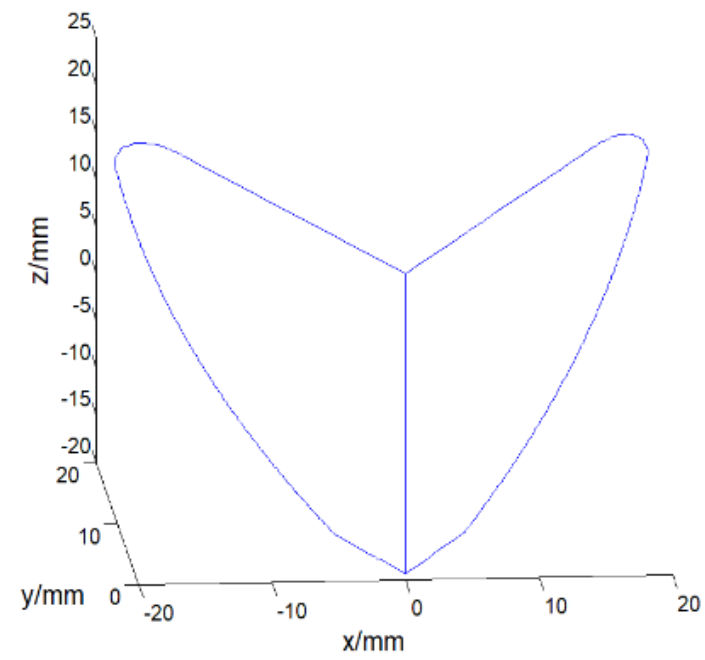

Figure 21: Boundaries trajectory.

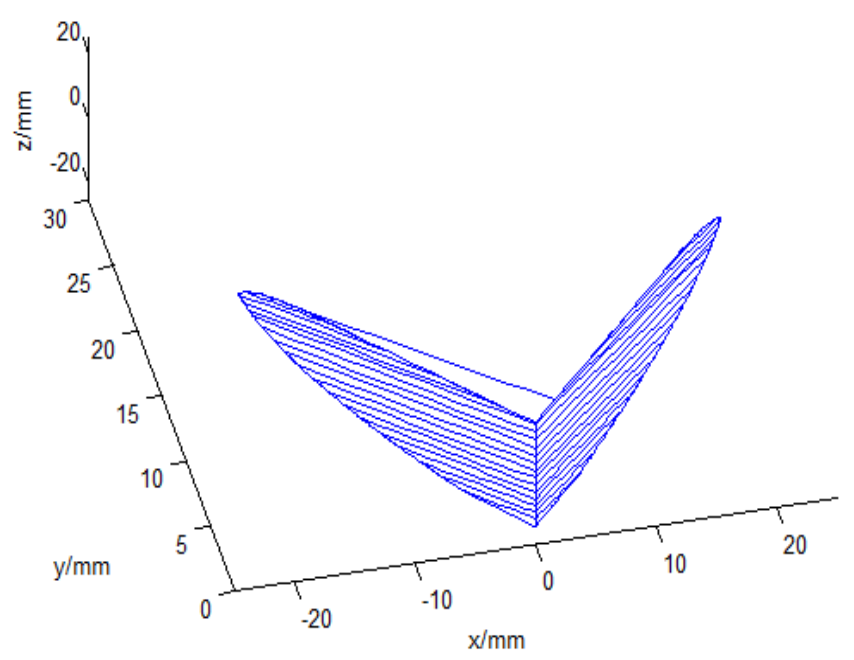

Figure 22: Internal and boundary synthesis trajectories.

The planes $\alpha$ and $\beta$ of the implant were cut vertically by the robot to the position $z=-19.405+n_{i} \cdot \alpha_{e o}$, leaving a V-shaped trajectory for femur grinding. Considering the radius of the ball cutting tool $r$, the burr height and grinding spacing were set to $h=0.16 \mathrm{~mm}$ and $a_{e o}=2.24 \mathrm{~mm}$, respectively, to ensure the flatness of the femoral surface. Figs. 20 and 21 show the simulated internal trajectory and boundaries of the implant. Fig. 22 presents the synthesized grinding trajectory. Due to the thickness of the implant, the grinding was carried out layer by layer until the shape matched the implant.

\section{CONCLUSIONS}

This paper designs a knee joint grinding robot with a planar parallel mechanism and a vertical mechanism. The inverse kinematics equation and forward kinematics equation of the robot were derived by the vector method. Then, the Monte-Carlo method was adopted to identify the workspace of the robot. After that, the author explained the basic procedure of robotassisted TKA and introduced the grinding trajectory planning based on the implant selected for the patient. By the planning method, the plane and boundary equations of the implant were determined; then, the femur was ground into the desired shape; after that, the implant was cut off with a ball cutting tool vertically to the depth equal to the row spacing. To validate the trajectory planning method, a simulation analysis was performed on the planned trajectory. The results prove the feasibility and efficiency of the proposed method. The research findings shed new light on the robot-assisted TKA.

\section{ACKNOWLEDGEMENT}

The authors would like to express appreciation to financial supports from Project funded by China Postdoctoral Science Foundation (2016M602164), and Project funded by Qingdao Postdoctoral Researchers Applied Research Foundation (2016119).

\section{REFERENCES}

[1] Liow, M. H. L.; Chin, P. L.; Tay, K. J. D.; Chia, S. L.; Lo, N. N.; Yeo, S. J. (2014). Early experiences with robot-assisted total knee arthroplasty using the DigiMatch $^{\mathrm{TM}}$ ROBODOC surgical system, Singapore Medical Journal, Vol. 55, No. 10, 529-534, doi:10.11622/ smedj.2014136

[2] Petermann, J.; Kober, R.; Heinze, J.; Frolich, J. J.; Heeckt, P. F.; Gotzen, L. (2000). Computerassisted planning and robot-assisted surgery in anterior cruciate ligament reconstruction, 
Operation Techniques in Orthopaedics, Vol. 10, No. 1, 50-55, doi:10.1016/s1048$6666(00) 80042-7$

[3] Jakopec, M.; Harris, S. J.; Rodriguez-y-Baena, F.; Gomes, P.; Davies, B. L. (2003). The Acrobot ${ }^{\circledR}$ system for total knee replacement, Industrial Robot, Vol. 30, No. 1, 61-66, doi:10.1108/01439910310457724

[4] Zhang, S.; Cao, D.; Li, S.; Min, H.; Feng, F. (2018). Inverse kinematic tension analysis and optimal design of a cable-driven parallel-series hybrid joint towards wheelchair-mounted robotic manipulator, Journal Européen des Systèmes Automatisés, Vol. 51, No. 1-3, 59-74, doi:10.3166/JESA.51.59-74

[5] Wang, Z.; Wang, G.; Yao, C. (2018). Robot path planning based on TGSA and three-order Bezier curve, Revue d'Intelligence Artificielle, Vol. 32, No. 1, 41-56, doi:10.3166/RIA.32.S1.41-56

[6] Kwon, D.-S.; Yoon, Y.-S.; Lee, J.-J.; Ko, S.-Y.; Huh, K.-H.; Chung, J.-H.; Park, Y.-B.; Won, C.H. (2001). ARTHROBOT: a new surgical robot system for total hip arthroplasty, Proceedings of the 2001 IEEE/RSJ International Conference on Intelligent Robots and Systems, 1123-1128, doi:10.1109/IROS.2001.976319

[7] Wolf, A.; Jaramaz, B.; Lisien, B.; DiGioia, A. M. (2005). MBARS: mini bone-attached robotic system for joint arthroplasty, International Journal of Medical Robotics and Computer Assisted Surgery, Vol. 1, No. 2, 101-121, doi:10.1002/rcs.20

[8] Bruni, S.; Cerveri, P.; Espinosa, I. (2007). An application of a hybrid robot in the total knee replacement procedure, Proceedings of the $12^{\text {th }}$ IFToMM World Congress on the Theory of Machines and Mechanisms, 8 pages

[9] Song, S.; Mor, A.; Jaramaz, B. (2009). HyBAR: hybrid bone-attached robot for joint arthroplasty, The International Journal of Medical Robotics and Computer Assisted Surgery, Vol. 5, No. 2, 223-231, doi:10.1002/rcs.254

[10] Abraham, N.; Wolf, A.; Choset, H. (2006). A potential function approach to surface coverage for a surgical robot, Computer Aided Surgery, Vol. 11, No. 1, 1-9, doi:10.3109/10929080500432173

[11] Guven, Y.; Barkana, D. E. (2010). Bone cutting trajectory generation using a medical user interface of an orthopedical surgical robotic system, Proceedings of the $3^{\text {rd }}$ Conference on Human System Interactions, 325-330, doi:10.1109/HSI.2010.5514548

[12] Sugita, N.; Nakano, T.; Abe, N.; Fujiwara, K.; Ozaki, T.; Suzuki, M.; Mitsuishi, M. (2011). Tool path strategy based on geometric model for multi-axis medical machine tool, CIRP Annals, Vol. 60, No. 1, 419-424, doi:10.1016/j.cirp.2011.03.030

[13] Fan, L.; Sun, L.; Du, Z. (2009). A tool-path interpolation algorithm based on unfolding-bending parametric curves, Key Engineering Materials, Vol. 407-408, 220-224, doi:10.4028/ www.scientific.net/KEM.407-408.220

[14] Tian, H.; Wang, C.; Dang, X.; Sun, L. (2017). A 6-DOF parallel bone-grinding robot for cervical disc replacement surgery, Medical \& Biological Engineering \& Computing, Vol. 55, No. 12, 2107-2121, doi:10.1007/s11517-017-1648-4

[15] Federspil, P. A.; Geisthoff, U. W.; Henrich, D.; Plinkert, P. K. (2003). Development of the first force-controlled robot for otoneurosurgery, The Laryngoscope, Vol. 113, No. 3, 465-471, doi:10.1097/00005537-200303000-00014 